\title{
Watching a Virus Expand
}

\author{
Bacteria-infecting viruses provide a controllable platform to study the \\ expansion of a virus in a cell population.
}

By Matteo Rini

W

hen viruses or bacteria infect an organism, the outcome of the infection depends on how they spread in their host. Researchers have devised many techniques for imaging bacterial expansion. Studying viruses, however, is trickier because, unlike bacteria, they can't survive without the host. An experimental platform for studying viral expansion must be able to probe the complex virus-host interactions. A team led by Diana Fusco of the University of Cambridge in the UK has now developed such a platform and applied it to study the waves of viral expansion in an infected cell population [1].

Using optical microscopy, the team observed how a bacteriophage-a virus that infects bacteria-colonized a droplet containing E. coli. Combining experiments with simulations, they analyzed the waves of viral expansion in the droplet. According to previous models, such waves should be "pulled," that is, driven by virus particles at the boundaries of the region the virus is invading. But the team found that the simulations that best fit the data involved "pushed" waves. In pushed waves, the wave front moves more slowly than the rest of the wave, meaning that particles within the infected region,

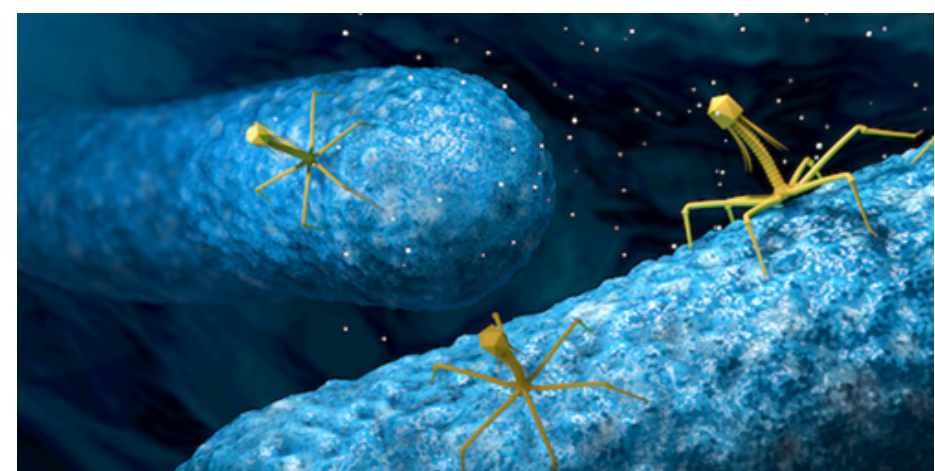

Credit: Christoph Burgstedt/stock.adobe.com rather than those at the periphery, are those that drive the expansion.

The result is surprising, as pushed waves are known to emerge in the presence of cooperative effects. Yet viruses don't interact directly with each other. The observation thus implies that an "effective cooperation" among viral particles arises indirectly through feedback mechanisms produced by the interaction of virus particles with their bacterial hosts. The researchers say that the realization that viruses expand by pushed waves will help in building better models for viral growth and evolution in infected organisms.

Matteo Rini is the Editor of Physics.

\section{REFERENCES}

1. M. Hunter et al., "Virus-host interactions shape viral dispersal giving rise to distinct classes of traveling waves in spatial expansions," Phys. Rev. X 11, 021066 (2021). 